\title{
Does microvascular invasion in Barcelona Clinic Liver Cancer stage A multinodular hepatocellular carcinoma indicate early- stage behavior?
}

\author{
Han Wang ${ }^{1,2,3 \#}$, Long-Hai Feng ${ }^{4 \#}$, You-Wen Qian ${ }^{1,2,3 \#}$, Zhen-Ying Cao ${ }^{1,2,3}$, Meng-Chao Wu ${ }^{5}$, \\ Wen-Ming Cong ${ }^{1,2,3}$
}

${ }^{1}$ Department of Pathology, Eastern Hepatobiliary Surgery Hospital, The Second Military Medical University, Shanghai 200438, China; ${ }^{2}$ Key Laboratory of Signaling Regulation and Targeting Therapy of Liver Cancer, The Second Military Medical Uneversity, Ministry of Education, Shanghai 200438, China; ${ }^{3}$ Key Laboratory of Hepatobiliary Tumor Biology, Eastern Hepatobiliary Surgery Hospital, Shanghai 200438, China; ${ }^{4}$ Department of Liver Surgery and Transplantation, Liver Cancer Institute and Zhongshan Hospital, Fudan University, The Key Laboratory for Carcinogenesis and Cancer Invasion, The Ministry of Education of China, Shanghai 200032, China; ${ }^{5}$ Department of Hepatic Surgery, Eastern Hepatobiliary Surgery Hospital, The Second Military Medical University, Shanghai 200438, China

Contributions: (I) Conception and design: WM Cong; (II) Administrative support: MC Wu; (III) Provision of study materials or patients: WM Cong, MC Wu; (IV) Collection and assembly of data: H Wang, LH Feng, YW Qian; (V) Data analysis and interpretation: H Wang, ZY Cao; (VI) Manuscript writing: All authors; (VII) Final approval of manuscript: All authors.

\#These authors contributed equally to this work.

Correspondence to: Wen-Ming Cong. Department of Pathology, Eastern Hepatobiliary Surgery Hospital, 225 Changhai Rd., Yangpu, Shanghai 200438, China. Email: wmcong@smmu.edu.cn.

Background: To identify the impact of tumor number on Barcelona Clinic Liver Cancer (BCLC) earlystage hepatocellular carcinoma (HCC) and the impact of microvascular invasion (MVI) on multinodular HCC (MHCC).

Methods: We retrospectively analyzed 1,548 patients who had early-stage HCC [solitary HCC (SHCC, $\mathrm{n}=1,481)$ and MHCC $(\mathrm{n}=67)]$, according to the BCLC classification, after curative resection. Recurrencefree survival (RFS) and overall survival (OS) were compared. Propensity score matching (PSM) was used to balance potential confounding factors.

Results: Both before and after PSM, significant differences were noted between the MHCC group and the SHCC group in RFS but not in OS. For the PSM cohort, the 5-year RFS rates were $7.5 \%$ and $41.2 \%$ for the MVI-positive MHCC group and the SHCC group, respectively $(\mathrm{P}<0.001)$. The 5 -year OS rates were $48.9 \%$ and $75.2 \%$ for the MVI-positive MHCC group and the SHCC group, respectively ( $\mathrm{P}=0.017)$. The RFS and OS were not significantly different between the MVI-negative MHCC group and the SHCC group. MVI $(\mathrm{P}=0.029)$ and multiple nodules $(\mathrm{P}=0.029)$ were associated with early recurrence.

Conclusions: The presence of MVI in BCLC early-stage MHCC was highly suggestive of a poor prognosis and should not be classified as early-stage biological behavior.

Keywords: Hepatocellular carcinoma (HCC); Barcelona Clinic Liver Cancer (BCLC); early stage; microvascular invasion (MVI); multiple nodules

Submitted June 20, 2019. Accepted for publication Aug 21, 2019.

doi: 10.21037/atm.2019.08.114

View this article at: http://dx.doi.org/10.21037/atm.2019.08.114 


\section{Introduction}

Hepatocellular carcinoma (HCC) is one of the most common causes of cancer-related deaths worldwide $(1,2)$. To date, numerous HCC staging systems have been proposed to classify patients for better prognostic prediction and treatment decisions $(3,4)$. Among these classification systems, the Barcelona Clinic Liver Cancer (BCLC) classification is the most widely used system and is recommended by the European Association for the Study of the Liver (EASL) and the American Association for the Study of Liver Diseases (AASLD) (5-7). Radiofrequency ablation, surgical resection and liver transplantation are regarded as curative treatment methods and are recommended for patients with BCLC early-stage (Stage 0 and Stage A) disease (8). Multinodular HCC (MHCC) with up to 3 nodules, each less than $3 \mathrm{~cm}$ in size, but without major vascular invasion and extrahepatic metastasis was also divided into early stages, and patients with MHCC are suggested for liver transplantation (9). However, since transplantation is limited by donor organ availability, surgical resection remains an appropriate choice for these patients $(10,11)$.

Recent progress has led to the recognition that both intrahepatic metastasis (IM) and multicentric occurrence (MO) contribute to multiple tumor nodules in the liver (12). IM-type HCC is accompanied by a worse biological behavior than MO-type HCC and does not benefit from curative therapy $(13,14)$. In addition, a previous study demonstrated that tumor biology and the condition of the underlying liver were better prognostic factors than tumor size and should be given closer attention (15). As such, we hypothesized that the IM-type original pattern also exists in BCLC early-stage MHCC and represents a poor prognosis. Interestingly, microvascular invasion (MVI), which is identified as the presence of tumor emboli in a vascular space on microscopy, highly indicates the possibility of early recurrence and metastasis $(16,17)$. Numerous studies have shown meaningful associations between the presence of MVI and IM-type HCC $(13,18)$. Therefore, in this study, we aimed to analyze the clinicopathological data of BCLC early-stage HCC patients to explore the significance of tumor number and MVI on prognostic and biological behavior and to illustrate whether IM-type MHCC exists in these patients, which might enable clinicians to better assess whether patients are suitable for surgery and to implement reasonable pre- and post-operative management.

\section{Methods}

\section{Patients}

The consecutive patients who underwent hepatic resection for BCLC early-stage HCC from December 2009 to December 2010 at the Eastern Hepatobiliary Surgery Hospital were identified. The inclusion criteria were as follows: (I) HCC within solitary nodules or up to 3 multiple nodules, with no nodules $>3 \mathrm{~cm}$, (II) Child-Pugh A-B, (III) Eastern Cooperative Oncology Group (ECOG) score =0, and (IV) underwent curative resection. The exclusion criteria were as follows: (I) major vascular invasion, (II) extrahepatic metastasis, (III) preoperative anticancer treatments, and (IV) a previous history of other malignancies.

A 7-point baseline sampling protocol was performed to evaluate the pathological parameters (19). HCC was diagnosed by two pathologists in all cases. MVI was defined as the presence of tumor cells in a portal vein, hepatic vein, or large capsular vessel of the surrounding hepatic tissue lined by endothelium that was visible only on microscopy (19). The clonal origin of MHCC was evaluated by the comprehensive criteria pertaining to tumor grade and histological type (20). The reported diameter and capsule are based on the tumor with the largest diameter. The reported Edmondson-Steiner grade (III-IV or I-II) is the highest grade found in the specimen.

\section{Follow-up}

The patients were followed up once every 2 months in the first year and once every 3 months thereafter. The follow-up investigations consisted of ultrasonographic scans, computed tomography scans, or magnetic resonance imaging with serum alpha-fetoprotein (AFP). The study endpoints were recurrence-free survival (RFS) and overall survival (OS). RFS was calculated from the date of surgery to the date when recurrence was diagnosed. OS was defined as the duration between surgery and the last follow-up visit or HCC-related death. The patients were followed up for 84 months.

\section{Statistical analysis}

Continuous variables are expressed as the mean \pm standard deviation. Chi-squared tests or Fisher's exact tests were used to compare categorical data. Continuous data were analyzed with Student's $t$-tests or Mann-Whitney U tests, 
when appropriate. Survival analyses of RFS and OS were performed by the Kaplan-Meier method with the log-rank test. A Cox proportional hazards regression model was used to develop a multivariable model. Variables with a $\mathrm{P}$ value less than 0.1 in the univariable analysis were entered into the multivariable model. Logistic regression models were used to determine the predictors associated with the presence of MVI. Variables with a $P$ value less than 0.2 in the univariable analysis were entered into the multivariable model.

The effect of selection bias and confounding factors was reduced by using propensity score matching (PSM) (21). All variables with potential differences $(\mathrm{P}<0.2)$ were entered into the PSM model, including sex, aspartate aminotransferase (AST), alkaline phosphatase (ALP), white blood cells (WBCs), platelets (PLTs), international normalized ratio (INR), hepatitis B e antibody (HBeAb), use of Pringle maneuver, use of transfusion, capsule, and presence of cirrhosis. Considering the strong correlation between prothrombin time (PT) and INR, we selected INR for propensity matching. Furthermore, we did not include tumor diameter for propensity matching as BCLC earlystage solitary HCC (SHCC) was not bound by tumor size. A logistic regression analysis was performed using nearest neighbor matching to estimate the propensity score. The ratio for matching was established at 1:2 using a caliper width of 0.1 of the standard deviation of the logit of the propensity score. The absolute standardized difference was computed to assess the balance of matched variables and to confirm whether the values were lower than 0.1 (22). In addition, the discrimination of the propensity score model was assessed using the area under the receiver operating characteristic (ROC) curve. Calibration was assessed using the Hosmer-Lemeshow goodness-of-fit test. The HosmerLemeshow test compared model performance (observed $v s$. expected) across deciles of risk to test whether the model was biased (i.e., performed differently at the extremes of risk). A nonsignificant value for the Hosmer-Lemeshow test suggested an absence of such bias (23).

All $\mathrm{P}$ values were 2 tailed, and $\mathrm{P}<0.05$ was considered statistically significant. All statistical analyses were conducted with SPSS 24.0 (IBM, New York, USA) and R software (version 3.4.2, http://www.r-project.org/).

\section{Results}

\section{Baseline characteristics}

Our selection criteria identified 1,548 patients with BCLC early-stage HCC. Of these patients, 1,481 and 67 patients had SHCC and MHCC, respectively. PSM was performed to overcome the imbalances between these two groups and resulted in 126 patients with SHCC and 64 patients with MHCC. No significant differences existed between the two groups (all $\mathrm{P}>0.05$ ). The baseline characteristics of the patients are listed in Table 1. The effectiveness of PSM is shown in Table 1 and Figure S1. Figure S2 shows the correlation between MVI and the clonal origin pattern of MHCC.

\section{Risk factors for RFS and OS}

The survival analysis of the crude cohort is shown in Table 2. The multivariate Cox proportional hazards model identified a high total bilirubin (TBIL), high $\gamma$-glutamyl transpeptidase (GGT), high INR, HBV DNA load $>10^{3} \mathrm{IU} / \mathrm{mL}$, positive hepatitis $\mathrm{B}$ e antigen ( $\mathrm{HBeAg}$ ), large tumor size, multiple tumor nodules, and MVI as risk factors for RFS. A high TBIL, low albumin (ALB), high GGT, high AFP, positive HBeAg, large tumor size, presence of cirrhosis, poor tumor differentiation and MVI remained independent risk factors for poor OS. Table S1 shows the Cox regression analysis of the PSM cohort.

\section{Impact of tumor number before and after PSM}

Before PSM, the 1-, 3-, 5-, and 7-year RFS rates in the SHCC group were $71.3 \%, 52.9 \%, 44.3 \%$, and $38.0 \%$ and those in the MHCC group were $65.9 \%, 41.2 \%, 29.6 \%$, and $24.7 \%$, respectively (Figure $1 A, \mathrm{P}=0.012$ ). The mean RFS of the SHCC and MHCC groups were 45.71 and 35.57 months, respectively. The 1-, 3-, 5-, and 7-year OS rates of the SHCC group were $91.7 \%, 79.2 \%, 70.0 \%$, and $63.8 \%$ and those of the MHCC group were $86.3 \%, 75.4 \%$, $65.9 \%$, and $61.1 \%$, respectively (Figure $1 B, \mathrm{P}=0.573$ ). The mean OS of the SHCC and MHCC groups were 65.76 and 63.06 months, respectively. After PSM, the 1-, 3-, 5-, and 7 -year RFS rates of the SHCC group were $74.2 \%, 54.6 \%$, $41.2 \%$ and $36.1 \%$ and those of the MHCC group were $64.2 \%, 39.9 \%, 27.8 \%$, and $22.6 \%$, respectively (Figure 1C, $\mathrm{P}=0.029)$. The mean RFS of the SHCC group was 45.00 months compared with 34.29 months of the MHCC group. The 1-, 3-, 5-, and 7-year OS rates of the SHCC group were $93.6 \%, 85.5 \%, 75.2 \%$, and $63.2 \%$ and those of the MHCC group were $85.7 \%, 74.2 \%, 64.2 \%$, and $60.9 \%$, respectively (Figure $1 D, \mathrm{P}=0.493$ ). The mean $\mathrm{OS}$ of the SHCC and MHCC groups were 68.74 and 62.30 months, 
Table 1 Baseline characteristics of BCLC early stage HCC patients before and after propensity score matching

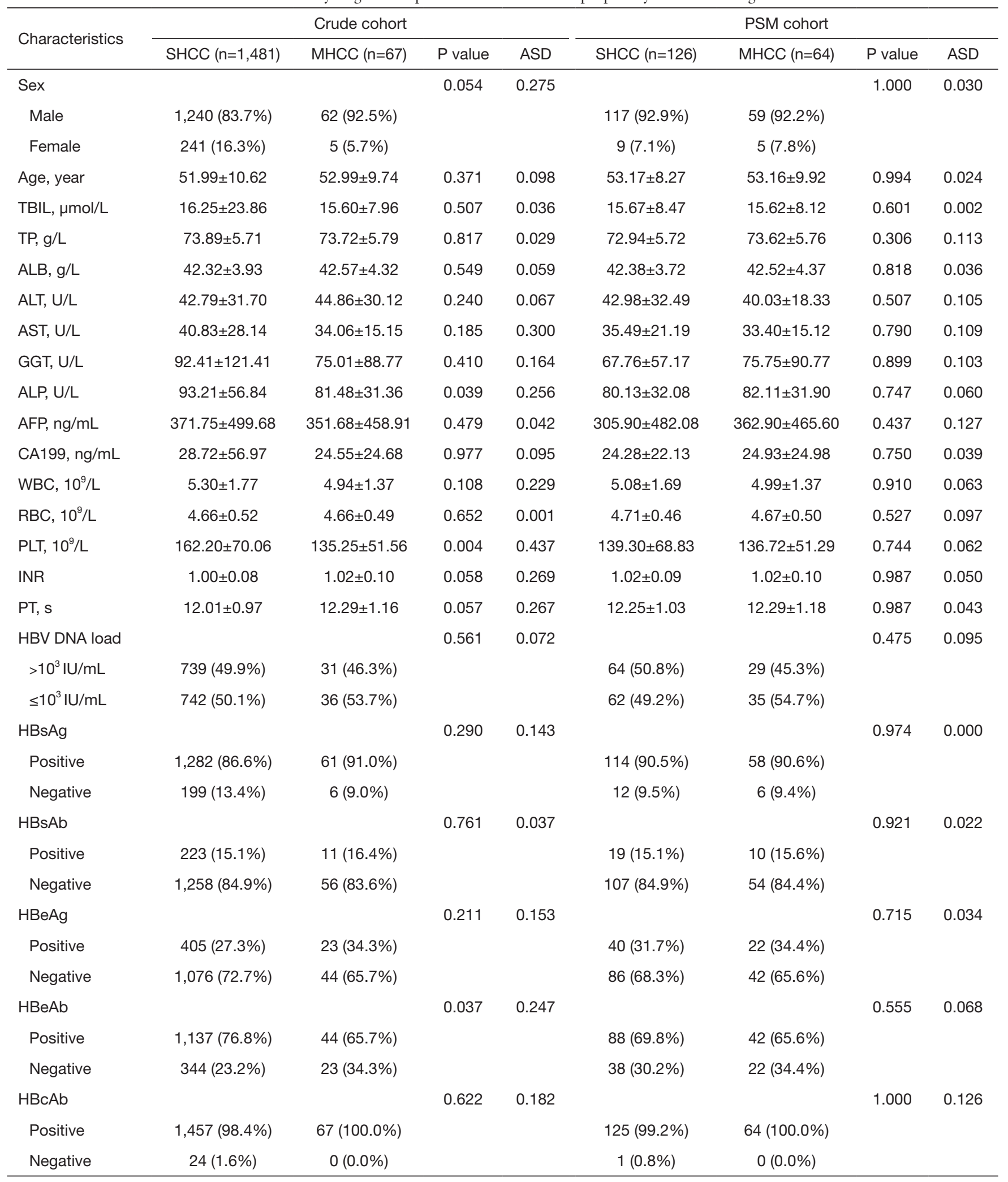

Table 1 (continued) 
Table 1 (continued)

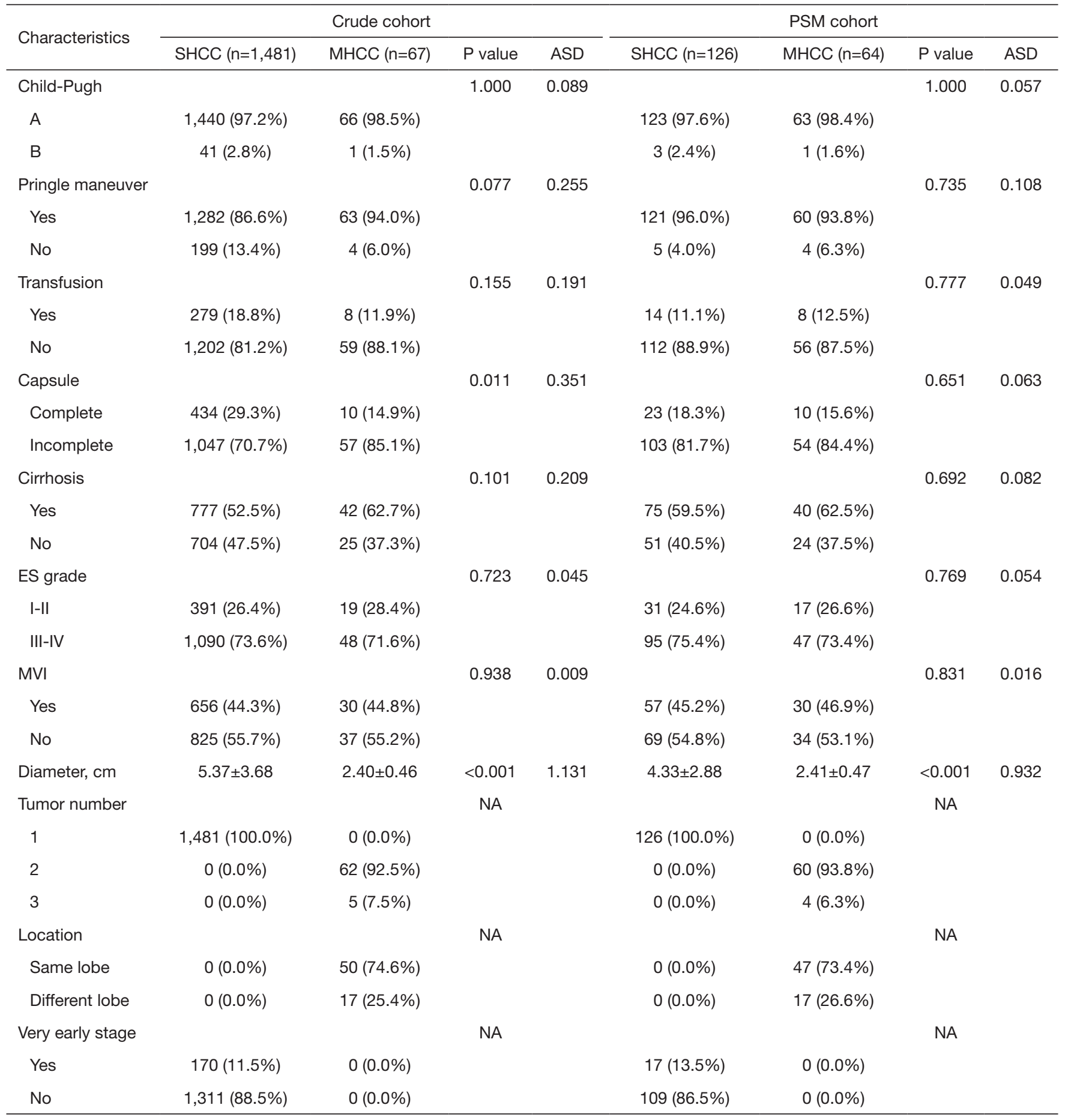

TBIL, total bilirubin; TP, total protein; ALB, albumin; ALT, alanine transaminase; AST, aspartate aminotransferase; GGT, $\gamma$-glutamyl transpeptidase; ALP, alkaline phosphatase; AFP, alpha fetal protein; CA199, carbohydrate antigen 19-9; WBC, white blood cells; RBC, red blood cells; PLT, platelets; INR, international normalized ratio; PT, prothrombin time; HBsAg, hepatitis B surface antigen; HBsAb, hepatitis B surface antibody; HBeAg, hepatitis B e antigen; HBeAb, hepatitis B e antibody; HBcAb, hepatitis B core antibody; ES, Edmondson-Steiner; MVI, microvascular invasion; NA, no answer; ASD, absolute standard difference. 


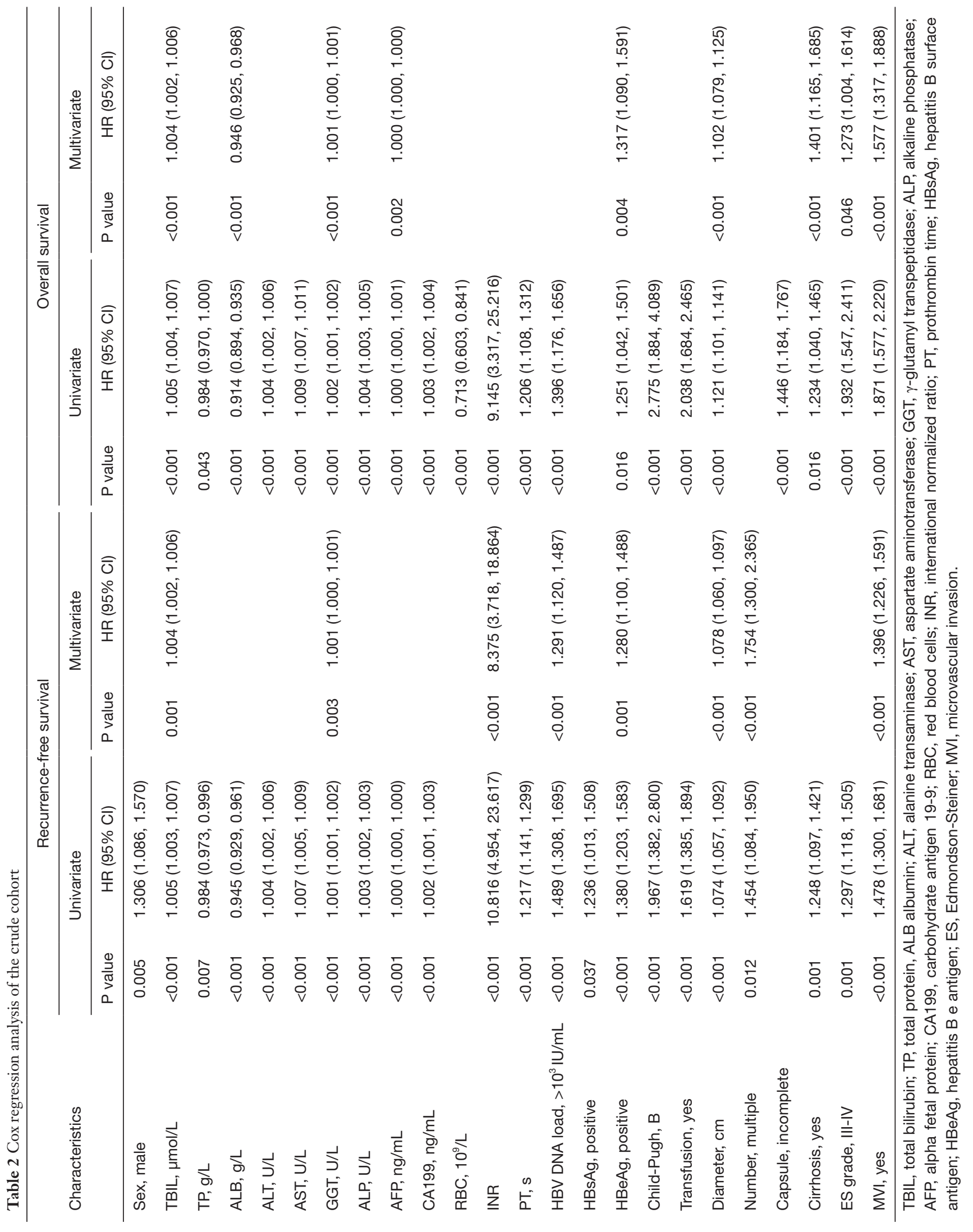



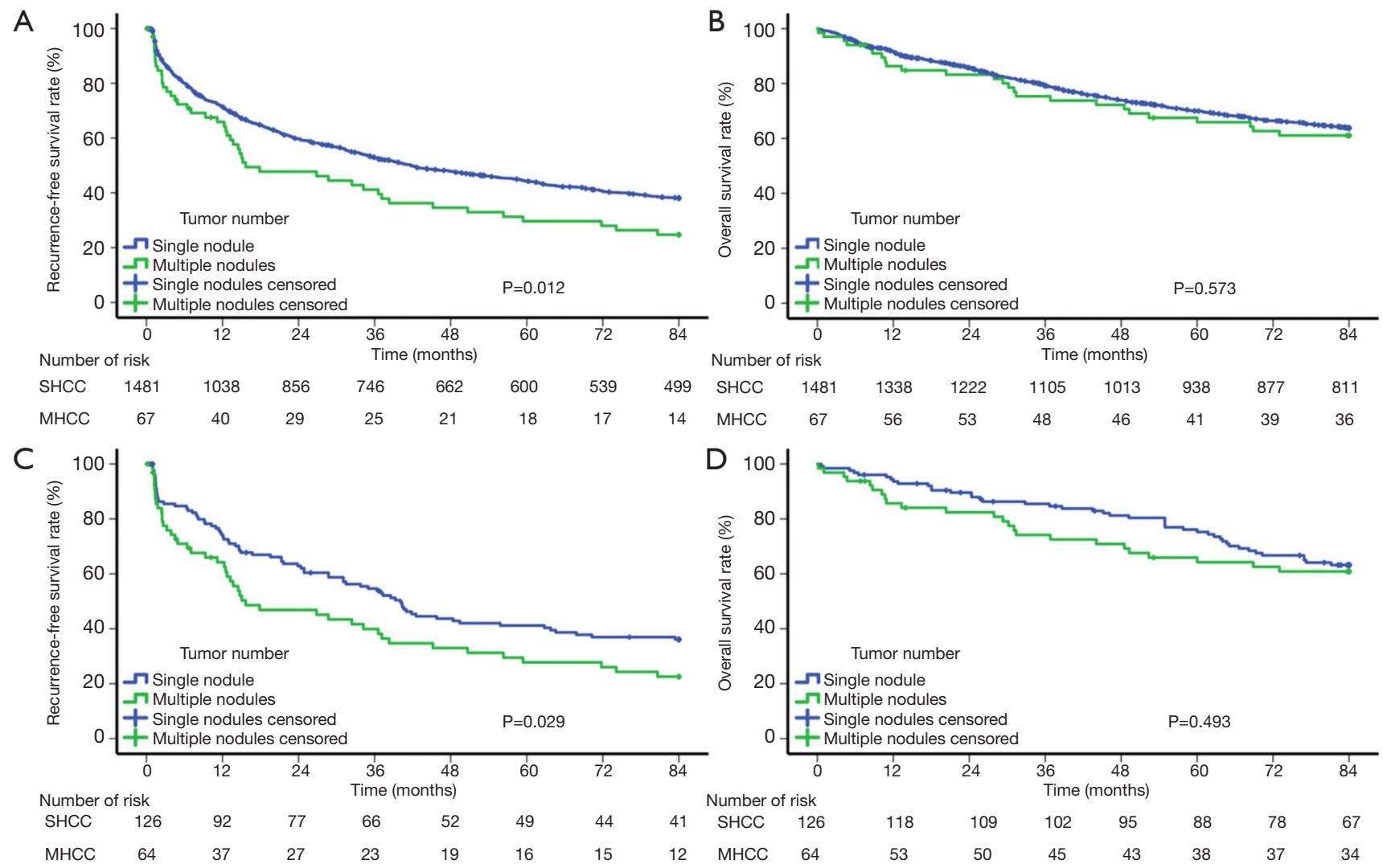

Figure 1 Cumulative incidence of recurrence-free survival and overall survival curves showing a comparison between SHCC patients and MHCC patients before (A,B) and after (C,D) propensity score matching. SHCC, solitary hepatocellular carcinoma; MHCC, multinodular hepatocellular carcinoma.

respectively.

\section{Subgroup analyses based on tumor number and MVI}

For all patients selected for the PSM cohort, the mean RFS and OS of the SHCC group, the MVI-negative MHCC group, and the MVI-positive MHCC group were 45.00, 45.46, and 21.70 months as well as $68.74,71.77$, and 52.05 months, respectively. The 1-, 3-, 5-, and 7-year RFS rates of the SHCC group were $74.2 \%, 54.6 \%, 41.2 \%$, and $36.1 \%$, those of the MVI-negative MHCC group were $68.5 \%, 52.2 \%$, $45.7 \%$, and $39.1 \%$, and those of the MVI-positive MHCC group were $59.6 \%, 26.1 \%, 7.5 \%$, and $3.7 \%$, respectively. The 1-, 3-, 5-, and 7-year OS rates of the SHCC group were $94.4 \%, 85.5 \%, 75.2 \%$, and $63.2 \%$, those of the MVInegative MHCC group were $94.1 \%, 87.8 \%, 78.3 \%$, and $75.0 \%$, and those of the MVI-positive MHCC group were $76.7 \%, 59.4 \%$, $48.9 \%$, and $45.4 \%$, respectively. The MVIpositive MHCC group had a worse RFS and OS than the SHCC group (RFS $\mathrm{P}<0.001$, Figure $2 A$; OS $\mathrm{P}=0.017$, Figure $2 B$ ). Nevertheless, the RFS and OS were not significantly different between the MVI-negative MHCC group and the SHCC group (RFS $\mathrm{P}=0.917$, Figure $2 A$; OS $\mathrm{P}=0.272$, Figure $2 B$ ). The predictors of MVI in the MHCC group are shown in Table $S 2$.

\section{Risk factors for early and late recurrence}

The independent risk factors for early tumor recurrence ( $<2$ years) were analyzed among all 190 patients in the PSM cohort, while the factors associated with late recurrence were assessed among the 104 patients who had a postoperative period of at least 2 years and did not have early recurrence (Table 3). The multivariate analysis demonstrated that multinodular tumors $[\mathrm{P}=0.029$, hazard ratio (HR) 1.655 ; 95\% confidence interval (CI), 1.0532.602] and MVI ( $\mathrm{P}=0.029$, HR 1.646; 95\% CI, 1.053-2.572) were associated with early recurrence. A high ALT $(\mathrm{P}=0.045$, 


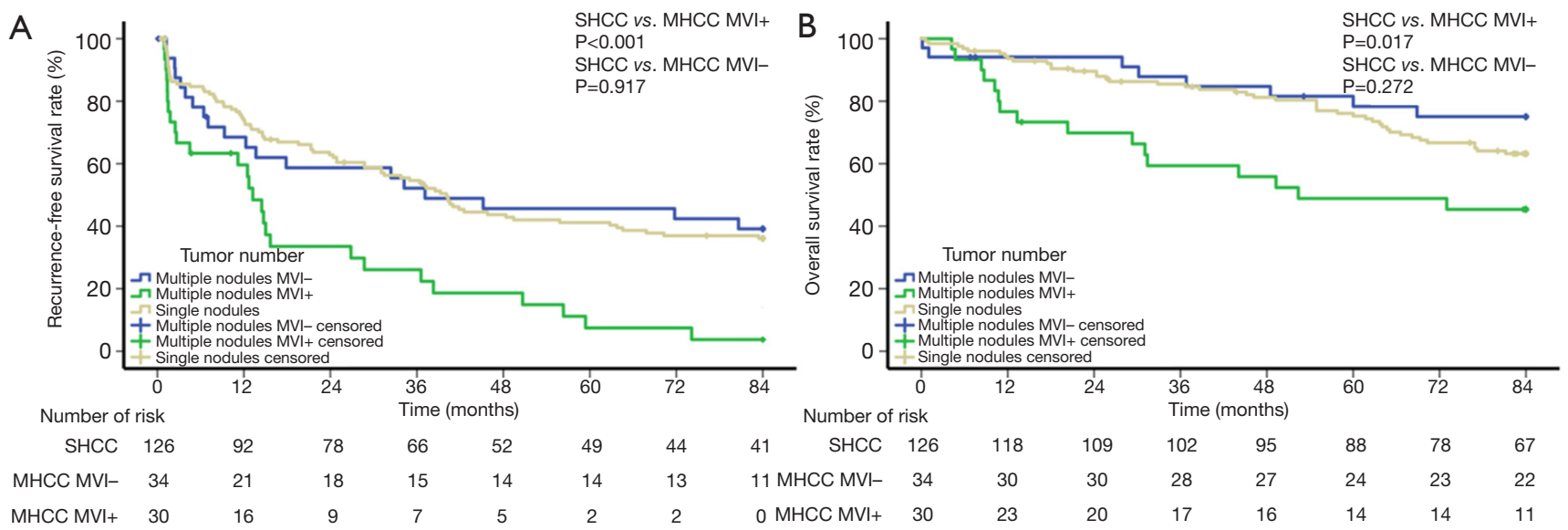

Figure 2 Cumulative incidences of recurrence-free survival (A) and overall survival (B) curves showing comparisons between SHCC patients and MHCC patients with/without MVI after propensity score matching. SHCC, solitary hepatocellular carcinoma; MHCC, multinodular hepatocellular carcinoma; MVI, microvascular invasion.

Table 3 Risk factors of early and late tumor recurrence

\begin{tabular}{|c|c|c|c|c|c|c|c|c|}
\hline \multirow{3}{*}{ Characteristics } & \multicolumn{4}{|c|}{ Early tumor recurrence $(n=190)$} & \multicolumn{4}{|c|}{ Late tumor recurrence $(n=104)$} \\
\hline & \multicolumn{2}{|r|}{ Univariate } & \multicolumn{2}{|r|}{ Multivariate } & \multicolumn{2}{|r|}{ Univariate } & \multicolumn{2}{|r|}{ Multivariate } \\
\hline & $P$ value & $\mathrm{HR}(95 \% \mathrm{Cl})$ & $P$ value & $\mathrm{HR}(95 \% \mathrm{Cl})$ & $P$ value & $\mathrm{HR}(95 \% \mathrm{Cl})$ & P Value & $\mathrm{HR}(95 \% \mathrm{Cl})$ \\
\hline ALT, U/L & & & & & 0.043 & $1.012(1.000,1.024)$ & 0.045 & $1.012(1.000,1.024)$ \\
\hline AST, U/L & & & & & 0.001 & $1.028(1.012,1.045)$ & & \\
\hline ALP, U/L & 0.001 & $1.008(1.003,1.013)$ & & & & & & \\
\hline AFP, $n g / m L$ & $<0.001$ & $1.001(1.000,1.001)$ & & & & & & \\
\hline $\mathrm{PLT}, 10^{\circ} / \mathrm{L}$ & 0.094 & $1.003(1.000,1.006)$ & & & & & & \\
\hline $\begin{array}{l}\text { Capsule, } \\
\text { incomplete }\end{array}$ & 0.015 & $2.613(1.201,5.684)$ & & & & & & \\
\hline ES grade, III-IV & 0.021 & $2.016(1.111,3.659)$ & & & & & & \\
\hline MVI, yes & 0.030 & $1.642(1.050,2.566)$ & 0.029 & $1.646(1.053,2.572)$ & 0.028 & $1.914(1.073,3.414)$ & & \\
\hline
\end{tabular}

ALT, alanine transaminase; AST, aspartate aminotransferase; GGT, $\gamma$-glutamyl transpeptidase; ALP, alkaline phosphatase; AFP, alpha fetal protein; PLT, platelets; ES, Edmondson-Steiner; MVI, microvascular invasion. 
HR 1.012; 95\% CI, 1.000-1.024) and large tumor size $(\mathrm{P}=0.027$, HR 1.132; 95\% CI, 1.014-1.264) were significant risk factors for late recurrence. For SHCC patients, the 6-month, 1-, and 2-year RFS rates were 84.7\%, 74.2\%, and $62.8 \%$, respectively; for MHCC patients, the $6-\mathrm{month}$, 1 -, and 2-year RFS rates were $71.0 \%, 64.2 \%$, and $46.8 \%$, respectively $(\mathrm{P}=0.028)$ (Figure 3). The recurrence pattern of MHCC is shown in Table $S 3$.

\section{Discussion}

Initially, when Llovet et al. proposed the BCLC staging classification, stage A4 (early stage) disease was defined as MHCC with up to 3 nodules smaller than $3 \mathrm{~cm}$, and other MHCCs were classified as stage B (intermediate stage) (5).

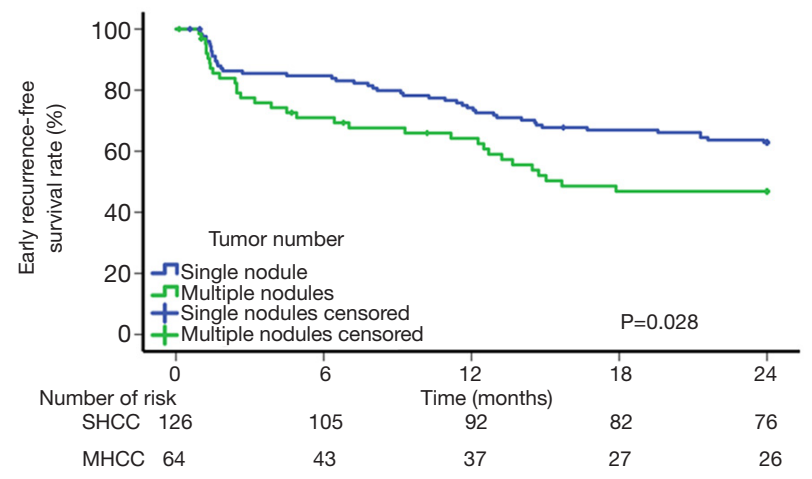

Figure 3 Cumulative incidence of early recurrence-free survival curve showing a comparison between SHCC patients and MHCC patients after propensity score matching. SHCC, solitary hepatocellular carcinoma; MHCC, multinodular hepatocellular carcinoma.
The authors believed that stage A4 HCC patients could achieve a beneficial prognosis through curative therapy, which was mainly referred to as the Milan criteria (9). However, the study that presented the Milan criteria included only 23 patients with MHCC, and all of the patients had unresectable MHCC; hence, the conclusion of the study might be biased. Furthermore, substantial studies have detected that multiple tumor nodules are important risk factors for early recurrence. For instance, $\mathrm{Li}$ et al. demonstrated that HCC patients classified with the Milan criteria achieved a poorer disease-free survival with an increased tumor number (24). Li et al. illustrated that multiple tumors were associated with early recurrence for patients who underwent $\mathrm{R} 0$ resection (25). These results compelled us to reappraise whether all BCLC early-stage MHCC patients were suitable for curative therapy. Namely, we considered that defining early-stage MHCC by only size and number of tumors was not rigorous. Combined with individualized and intensification treatments, the parameters that reflect the biological behaviors of tumor clearly offer the strongest evidence. Therefore, histopathological features have been the most valuable basis for retrospective explorations of the misjudgments of the BCLC staging classification.

One of the significant discoveries in the molecular pathology of HCC is the clonal origin pattern of MHCC (26). Two major clonal origin patterns of MHCC have been suggested; one model is the monoclonal origin of IM-type MHCC, and the other is the polyclonal origin of MO-type MHCC $(27,28)$. Among cases of MHCC, in cases where the tumor factors indicated high malignancy, the original tumor was hypothesized to lead to IM-type HCC, whereas MO-type $\mathrm{HCC}$ would be produced in other portions of the liver, such

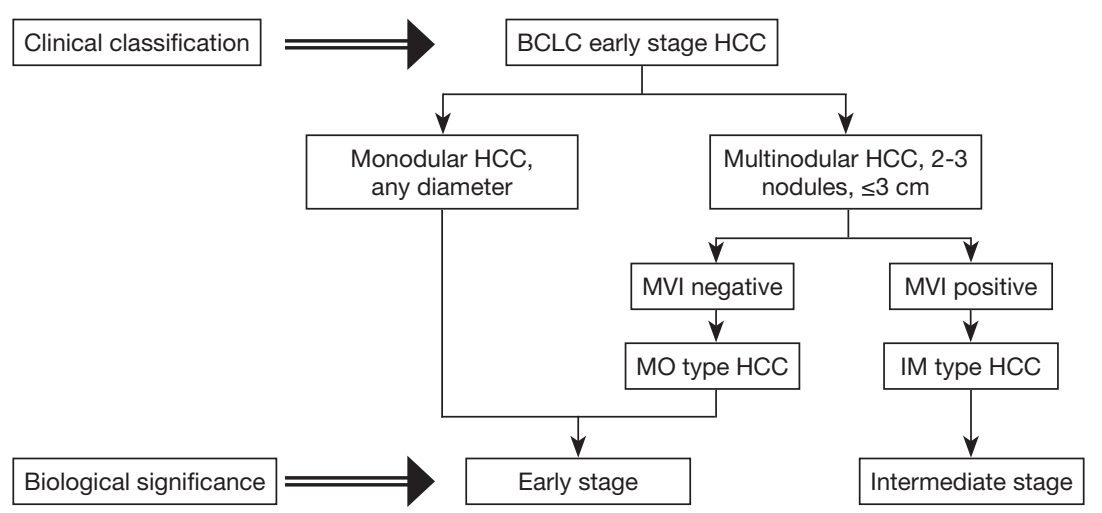

Figure 4 Schematic diagram of reappraisal for the BCLC early-stage patients. BCLC, Barcelona Clinic Liver Cancer. 
as in environments with poor background liver factors (29). Remarkably, MVI is a pathological phenomenon highly suggestive of early recurrence and unfavorable prognoses of HCC, and MVI is also a pathological factor highly correlated with IM of HCC (30). In our previous study of the clonal origin analysis of 40 recurrent HCCs, IMtype HCC had a higher frequency of vascular invasion than MO-type HCC (13). Kim et al. analyzed 198 MHCC patients and reported that MVI was the foremost factor for discriminating between the IM-type group and the MOtype group (18). Interestingly, our study also suggested a close correlation between MVI and IM-type MHCC. Since no consensus exists on the technology and criteria for determining the clonal origin of HCC, we believe that MVI represents malignant biological behavior and is a practical indicator for identifying IM-type MHCC.

The first observation of this study with a large cohort of patients with BCLC early-stage HCC was that the MHCC group had a higher recurrence rate than the SHCC group both before and after PSM. Furthermore, the presence of multiple tumor nodules was an independent risk factor for early recurrence but not late recurrence. These results prompted us to determine the possibility of earlystage MHCC forming IM and having MVI. Therefore, a subgroup analysis was conducted to explore the impact of MVI on the prognosis of MHCC. The Kaplan-Meier analysis implied that only MHCC patients without MVI had similar clinical outcomes to SHCC patients, while MHCC patients with MVI were associated with a decreased clinical outcome compared with SHCC patients. Considering the $44 \%$ detection rate of MVI, we believe that patients with BCLC early-stage HCC and MVI should not be classified as the same stage as those with SHCC and MHCC without MVI. The underlying cause is that MHCC with MVI is probably formed by IM, so the biological behavior of these tumors is highly malignant and therefore, these tumors are unable to benefit from curative treatment (Figure 4).

Current technology still cannot accurately predict MVI preoperatively, which is an inevitable limitation of our study. Notably, some research has proposed some efficient prediction models for MVI (31-33). Thus, we also created a prediction model for MVI based on our data to provide a reference for treatment selection for BCLC early-stage MHCC patients. More accurate prediction models for MVI for these patients are anticipated in the future.

To the best of our knowledge, our study was the first to analyze the impact of tumor number on BCLC earlystage patients and to suggest the role of MVI in MHCC.
Moreover, we used PSM to balance the baseline data of the included patients, thus avoiding interference bias from other factors on the prognosis (21). Furthermore, the detection rate of MVI in our study was more authentic than that in other studies because a 7-point baseline sampling protocol was performed for all specimens. Tissue specimens sampled at the junction of the tumor and the adjacent liver tissues can typically reflect the parameters that indicate biological tumor behaviors such as MVI, capsule, and grade.

Some limitations exist in our study; first, our study was a single-center study. Considering the different treatment experiences of different hospitals, multicenter research is still essential. Second, the number of BCLC early-stage MHCC patients in our study was relatively small, and although PSM could overcome bias to a certain extent, our conclusions still need to be verified in a larger cohort. In addition, liver transplantation is also a selective treatment option for BCLC early-stage MHCC, but these patients were valuable to the analyses of our study.

\section{Conclusions}

The presence of MVI in BCLC early-stage MHCC was highly suggestive of poor postoperative outcomes, which is possibly due to the malignant biological behavior of tumors caused by IM. The BCLC staging system should reappraise MHCC based on parameters that reflect the presence of MVI and distinguished the biological behavior level of early-stage MHCC.

\section{Acknowledgments}

Funding: We have received funding from the National Natural Science Foundation of China (Grant No. 81472278 and No. 81502086), the Funds for Creative Research Groups of National Natural Science Foundation of China (Grant No. 81521091), and the Scientific Research Foundation of Shanghai Municipal Commission of Health and Family Planning (Grant No. 20154Y0140) in this article.

\section{Footnote}

Conflicts of Interest: The authors have no conflicts of interest to declare.

Ethical Statement: The authors are accountable for all aspects of the work in ensuring that questions related 
to the accuracy or integrity of any part of the work are appropriately investigated and resolved. This study was conducted in accordance with the Declaration of Helsinki and approved by the Clinical Research Ethics Committee of the Eastern Hepatobiliary Surgery Hospital of Shanghai, China (No. EHBHKY2015-02-001). Informed consent was obtained from all patients for their dates to be used for research.

\section{References}

1. Chen $\mathrm{W}$, Zheng R, Baade PD, et al. Cancer statistics in China, 2015. CA Cancer J Clin 2016;66:115-32.

2. Siegel RL, Miller KD, Jemal A. Cancer statistics, 2018. CA Cancer J Clin 2018;68:7-30.

3. Kitai S, Kudo M, Izumi N, et al. Validation of three staging systems for hepatocellular carcinoma (JIS score, biomarker-combined JIS score and BCLC system) in 4,649 cases from a Japanese nationwide survey. Dig Dis 2014;32:717-24.

4. Addissie BD, Roberts LR. Classification and staging of hepatocellular carcinoma: an aid to clinical decisionmaking. Clin Liver Dis 2015;19:277-94.

5. Llovet JM, Bru C, Bruix J. Prognosis of hepatocellular carcinoma: the BCLC staging classification. Semin Liver Dis 1999;19:329-38.

6. European Association For The Study Of The Liver; European Organisation For Research And Treatment Of Cancer. EASL-EORTC clinical practice guidelines: management of hepatocellular carcinoma. J Hepatol 2012;56:908-43.

7. Heimbach JK, Kulik LM, Finn RS, et al. AASLD guidelines for the treatment of hepatocellular carcinoma. Hepatology 2018;67:358-80.

8. Forner A, Reig M, Bruix J. Hepatocellular carcinoma. Lancet 2018;391:1301-14.

9. Mazzaferro V, Regalia E, Doci R, et al. Liver transplantation for the treatment of small hepatocellular carcinomas in patients with cirrhosis. N Engl J Med 1996;334:693-9.

10. Utsunomiya $T$, Shimada $M$, Taguchi KI, et al. Clinicopathologic features and postoperative prognosis of multicentric small hepatocellular carcinoma. J Am Coll Surg 2000;190:331-5.

11. Xie DY, Ren ZG, Zhou J, et al. Critical appraisal of Chinese 2017 guideline on the management of hepatocellular carcinoma. Hepatobiliary Surg Nutr 2017;6:387-96.
12. Cong WM, Wu MC. New insights into molecular diagnostic pathology of primary liver cancer: Advances and challenges. Cancer Lett 2015;368:14-9.

13. Wang B, Xia CY, Lau WY, et al. Determination of clonal origin of recurrent hepatocellular carcinoma for personalized therapy and outcomes evaluation: a new strategy for hepatic surgery. J Am Coll Surg 2013;217:1054-62.

14. Wang Z, Gong W, Shou D, et al. Clonal Origin of Hepatocellular Carcinoma and Recurrence After Liver Transplantation. Ann Transplant 2016;21:484-90.

15. Kluger MD, Salceda JA, Laurent A, et al. Liver resection for hepatocellular carcinoma in 313 Western patients: tumor biology and underlying liver rather than tumor size drive prognosis. J Hepatol 2015;62:1131-40.

16. Feng LH, Dong H, Lau WY, et al. Novel microvascular invasion-based prognostic nomograms to predict survival outcomes in patients after $\mathrm{R} 0$ resection for hepatocellular carcinoma. J Cancer Res Clin Oncol 2017;143:293-303.

17. Wang H, Wu MC, Cong WM. Microvascular invasion predicts a poor prognosis of solitary hepatocellular carcinoma up to $2 \mathrm{~cm}$ based on propensity score matching analysis. Hepatol Res 2019;49:344-54.

18. Kim JM, Kwon CH, Joh JW, et al. Intrahepatic metastasis is more risky than multiple occurrence in hepatocellular carcinoma patients after curative liver resection. Hepatogastroenterology 2015;62:399-404.

19. Cong WM, Bu H, Chen J, et al. Practice guidelines for the pathological diagnosis of primary liver cancer: 2015 update. World J Gastroenterol 2016;22:9279-87.

20. Oikawa T, Ojima H, Yamasaki S, et al. Multistep and multicentric development of hepatocellular carcinoma: histological analysis of 980 resected nodules. J Hepatol 2005;42:225-9.

21. Rubin DB, Thomas N. Matching using estimated propensity scores: relating theory to practice. Biometrics 1996;52:249-64.

22. Austin PC. Balance diagnostics for comparing the distribution of baseline covariates between treatment groups in propensity-score matched samples. Stat Med 2009;28:3083-107.

23. Tada T, Kumada T, Toyoda H, et al. Impact of the branched-chain amino acid to tyrosine ratio and branchedchain amino acid granule therapy in patients with hepatocellular carcinoma: A propensity score analysis. J Gastroenterol Hepatol 2015;30:1412-9.

24. Li J, Zhou J, Yang PH, et al. Nomograms for survival prediction in patients undergoing liver resection for 
hepatitis B virus related early stage hepatocellular carcinoma. Eur J Cancer 2016;62:86-95.

25. Li Z, Lei Z, Xia Y, et al. Association of Preoperative Antiviral Treatment With Incidences of Microvascular Invasion and Early Tumor Recurrence in Hepatitis B Virus-Related Hepatocellular Carcinoma. JAMA Surg 2018:e182721.

26. Nomoto S, Hishida M, Inokawa $Y$, et al. Management of hepatocellular carcinoma should consider both tumor factors and background liver factors. Hepatobiliary Surg Nutr 2014;3:82-5.

27. Hodges KB, Cummings OW, Saxena R, et al. Clonal origin of multifocal hepatocellular carcinoma. Cancer 2010;116:4078-85.

28. Chen G, Cai Z, Li Z, et al. Clonal evolution in long-term follow-up patients with hepatocellular carcinoma. Int J Cancer 2018;143:2862-70.

29. Gehrau R, Mas V, Archer KJ, et al. Molecular classification and clonal differentiation of hepatocellular carcinoma: the

Cite this article as: Wang H, Feng LH, Qian YW, Cao ZY, Wu MC, Cong WM. Does microvascular invasion in BCLC stage A multinodular hepatocellular carcinoma indicate earlystage behavior? Ann Transl Med 2019;7(18):428. doi: 10.21037/ atm.2019.08.114 step forward for patient selection for liver transplantation. Expert Rev Gastroenterol Hepatol 2011;5:539-52.

30. Wang H, Du PC, Wu MC, et al. Postoperative adjuvant transarterial chemoembolization for multinodular hepatocellular carcinoma within the Barcelona Clinic Liver Cancer early stage and microvascular invasion. Hepatobiliary Surg Nutr 2018;7:418-28.

31. Pote N, Cauchy F, Albuquerque M, et al. Performance of PIVKA-II for early hepatocellular carcinoma diagnosis and prediction of microvascular invasion. J Hepatol 2015;62:848-54.

32. Banerjee S, Wang DS, Kim HJ, et al. A computed tomography radiogenomic biomarker predicts microvascular invasion and clinical outcomes in hepatocellular carcinoma. Hepatology 2015;62:792-800.

33. Zheng J, Chakraborty J, Chapman WC, et al. Preoperative Prediction of Microvascular Invasion in Hepatocellular Carcinoma Using Quantitative Image Analysis. J Am Coll Surg 2017;225:778-88.e1. 


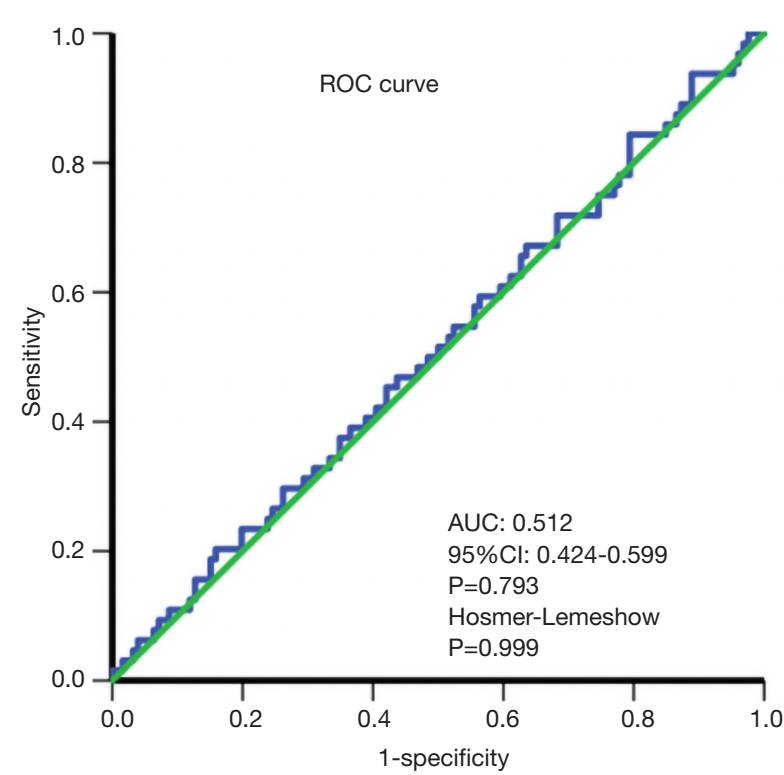

Figure S1 Evaluation of effectiveness about propensity score matching.

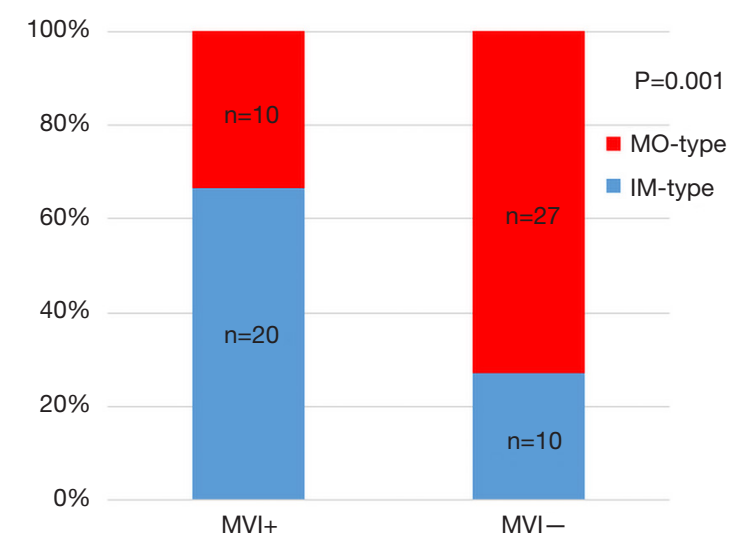

Figure S2 Correlation between microvascular invasion and clonal origin in early-stage MHCC patients. MHCC, multinodular hepatocellular carcinoma.

Table S1 Cox regression analysis of the propensity score matching cohort

\begin{tabular}{|c|c|c|c|c|c|c|c|c|}
\hline \multirow{3}{*}{ Characteristics } & \multicolumn{4}{|c|}{ Recurrence-free survival } & \multicolumn{4}{|c|}{ Overall survival } \\
\hline & \multicolumn{2}{|r|}{ Univariate } & \multicolumn{2}{|r|}{ Multivariate } & \multicolumn{2}{|r|}{ Univariate } & \multicolumn{2}{|r|}{ Multivariate } \\
\hline & $P$ value & $\mathrm{HR}(95 \% \mathrm{Cl})$ & $P$ value & $\mathrm{HR}(95 \% \mathrm{Cl})$ & $P$ value & $\mathrm{HR}(95 \% \mathrm{Cl})$ & $P$ value & $\mathrm{HR}(95 \% \mathrm{Cl})$ \\
\hline Sex, male & 0.037 & $2.593(1.059,6.350)$ & & & 0.087 & $3.417(0.837,13.956)$ & & \\
\hline ALB, $g / L$ & & & & & 0.031 & $0.934(0.878,0.994)$ & 0.009 & $0.916(0.858,0.978)$ \\
\hline ALT, U/L & 0.019 & $1.007(1.001,1.013)$ & & & & & & \\
\hline AST, U/L & 0.025 & $1.008(1.001,1.016)$ & & & 0.001 & $1.012(1.005,1.020)$ & 0.004 & $1.012(1.004,1.020)$ \\
\hline GGT, U/L & 0.001 & $1.004(1.002,1.006)$ & & & 0.045 & $1.003(1.000,1.005)$ & & \\
\hline ALP, U/L & 0.005 & $1.007(1.002,1.012)$ & & & 0.019 & $1.006(1.001,1.011)$ & & \\
\hline AFP, ng/mL & 0.012 & $1.000(1.000,1.001)$ & & & $<0.001$ & $1.001(1.000,1.001)$ & 0.029 & $1.001(1.000,1.001)$ \\
\hline INR & & & & & 0.033 & $18.805(1.258,281.059)$ & & \\
\hline PT, s & & & & & 0.03 & $1.283(1.024,1.608)$ & & \\
\hline HBV DNA load, $>10^{3} \mathrm{IU} / \mathrm{mL}$ & 0.080 & $1.371(0.963,1.952)$ & 0.024 & $1.520(1.057,2.187)$ & & & & \\
\hline Transfusion, yes & & & & & 0.015 & $2.117(1.156,3.875)$ & & \\
\hline Diameter, cm & 0.018 & $1.077(1.013,1.145)$ & $<0.001$ & $1.135(1.065,1.209)$ & 0.003 & $1.115(1.039,1.198)$ & & \\
\hline Tumor number, multiple & 0.030 & $1.498(1.040,2.158)$ & 0.001 & $2.003(1.345,2.984)$ & & & & \\
\hline ES grade, III-IV & 0.038 & $1.572(1.026,2.409)$ & & & & & & \\
\hline MVI, yes & 0.002 & $1.739(1.220,2.479)$ & 0.001 & $1.787(1.250,2.553)$ & $<0.001$ & $2.560(1.556,4.212)$ & $<0.001$ & $2.612(1.531,4.457)$ \\
\hline
\end{tabular}

ALB, albumin; ALT, alanine transaminase; AST, aspartate aminotransferase; GGT, $\gamma$-glutamyl transpeptidase; ALP, alkaline phosphatase; AFP, alpha fetal protein; INR, international normalized ratio; PT, prothrombin time; ES, Edmondson-Steiner; MVI, microvascular invasion. 
Table S2 Predictors of MVI for the early-stage MHCC

\begin{tabular}{lcccc}
\hline \multirow{2}{*}{ Characteristics } & \multicolumn{2}{c}{ Univariate } & & \multicolumn{2}{c}{ Multivariate } \\
\cline { 2 - 3 } \cline { 4 - 5 } TBIL, $\mu \mathrm{mol} / \mathrm{L}$ & P value & OR $(95 \% \mathrm{Cl})$ & P value $(95 \% \mathrm{Cl})$ \\
HBsAb, negative & 0.085 & $1.074(0.990,1.164)$ & & $21.506(2.073,223.126)$ \\
Capsule, no & 0.028 & $10.741(1.287,89.608)$ & 0.010 & $7.182(1.206,42.756)$ \\
AFP, ng/mL & 0.105 & $3.862(0.754,19.794)$ & 0.030 & $1.002(1.000,1.003)$ \\
\hline
\end{tabular}

MHCC, multinodular hepatocellular carcinoma; MVI, microvascular invasion; MHCC, multinodular hepatocellular carcinoma; TBIL, total bilirubin; HBsAb, hepatitis B surface antibody; AFP, alpha fetal protein.

Table S3 Recurrence pattern of the early-stage MHCC

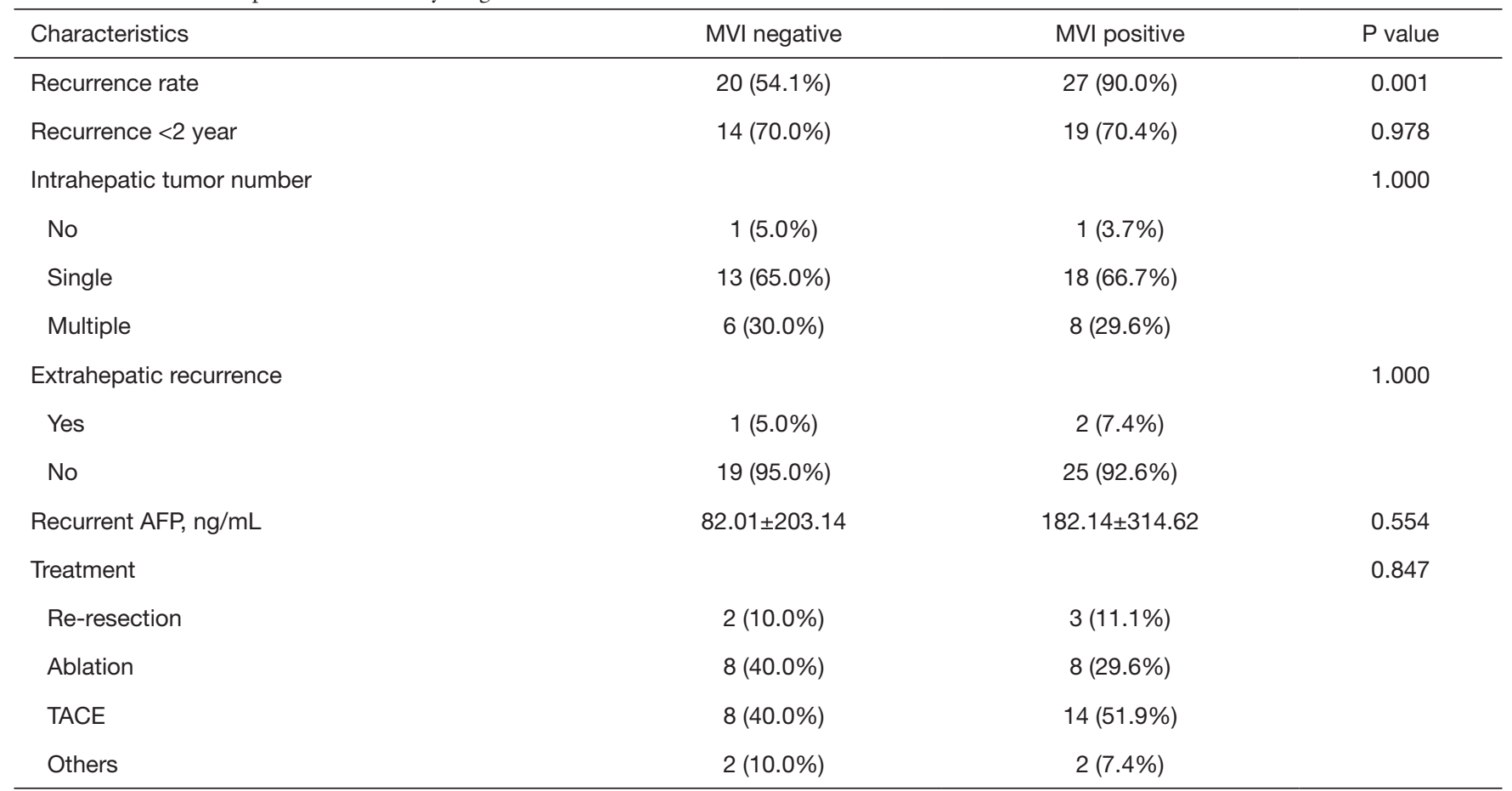

MHCC, multinodular hepatocellular carcinoma; AFP, alpha fetal protein; TACE, transcatheter arterial chemoembolization. 